Journal of Engineering and Applied Sciences 14 (6): 1770-1774, 2019

ISSN: 1816-949X

(C) Medwell Journals, 2019

\title{
Electromagnetic Field and Changing the Ambient Temperature of High Tension Wires
}

\author{
Adnan Salim Ahmad and Saad Izaldeen Majeed \\ Northern Technical University, \\ Technical Institute of Mosul, Mosul, Iraq
}

\begin{abstract}
In this research, we demonstrate the effect of changing ambient temperature on the intensity of the electromagnetic field generated near high voltage power transmission lines $(132 \mathrm{kV})$ in selected area South of Mosul city (Iraq), by measuring the increase in conductor length between two consecutive towers using electronic total station Leica TPS400 series. In the set of measurement cond uctor length changes from (30-80 $\mathrm{cm})$ and magnetic field intensity changes from $(33-44 \mathrm{mG})$ due to temperature changes range $\left(4-53^{\circ} \mathrm{C}\right)$. The calculated magnetic and electric field stages are compared to experience strategies given by International Commission on Non-Ionizing Radiation Protection (ICNIRP) to evaluate the risks. It has been noticed that the higher the ambient temperature around conductor, the more intense electromagnetic field which in turn leads to the lack of safe pasacclimatee this at constant load. As also noted, the less distance between two consecutive towers (SPAN) the greater the acclimate. So, these observations should be taken in account when design power transmissions line in those areas.
\end{abstract}

$\underline{\text { Key words: Electromagnetic field, transmission lines, conductor, observations, pasacclimatee, commission }}$

\section{INTRODUCTION}

Electricity is used to the profit of people wholly about the world. wheresoever electricity is transmitted, generated or used, electromagnetic fields (electric and magnetic domains) are shaped. These fields are a straight result of the attendance and or motion of electric charges. It is unbearable to generate and uacclimatee electrical energy without making these fields, hence they are an unavoidable result of our dependence on this form of energy (Pavlinic and Komen, 2017). Although, there is no sure recognized mechanism of how the electromagnetic fields at power frequency $(50 / 60 \mathrm{~Hz})$ can shake human health, it has been epidemiologically exposed that they have numerous hazards on human health. Where exposure of human form to electric field and magnetic field could reason biological effects, counting variations in functions of cells and tissues and delicate changes in hormone stages which may or can not be damaging. Also the power frequency Fields can delay with the close electrical and electronic equipment (Portier and Wolfe, 1998; Muhammad, 2011; Albizu et al., 2011).

To design a transmission line a certain details are desired as inputs for magnetic field calculation are of most important details. The vertical position of conductors (ground clearance) which varies with temperature which in turn vassal on a number of factors such as electrical load, ambient temperature and velocity as well as line layout design.
The design temperature and the minimum crushed permission are key issues in line design parameters. The conductor kind and size are significant in deciding the mechanical strength and the energy transfer competence of the line (Morgan, 1982).

The goal of this work to study the influence of changing the ambient temperature on electromagnetic fields at a certain point of transmission line $(132 \mathrm{kV})$ in Hammam al Allil Southern of Mosul city. Ground clearance measured at different temperatures using advanced surveying devices and electromagnetic fields were calculated at different distance from tower center then we are compared to exposure guidelines given by International Commission on Non-Ionizing Radiation Protection (ICNIRP) to evaluate the risks.

Background: The rating of conductor is affected by many factors which fall in two main branches. The first contains those factors which uncontrolled like weather conditions, conductor surface characteristic and solar irradiation. While the controlled factors contain the design parameters like the maximum conductor temperature and allowable loss of strength are the most significant. These two factors are interrelated, since, higher conductor temperature result in more rapid loss of strength (Anonymous, 1988, 2015). The traditional approach to rating conductor requires the selection of fixed ambient conditions and conductor temperature. The selection of most severe ambient conditions result in ratings which are

Corresponding Author: Adnan Salim Ahmad, Northern Technical University, Mosul, Iraq 


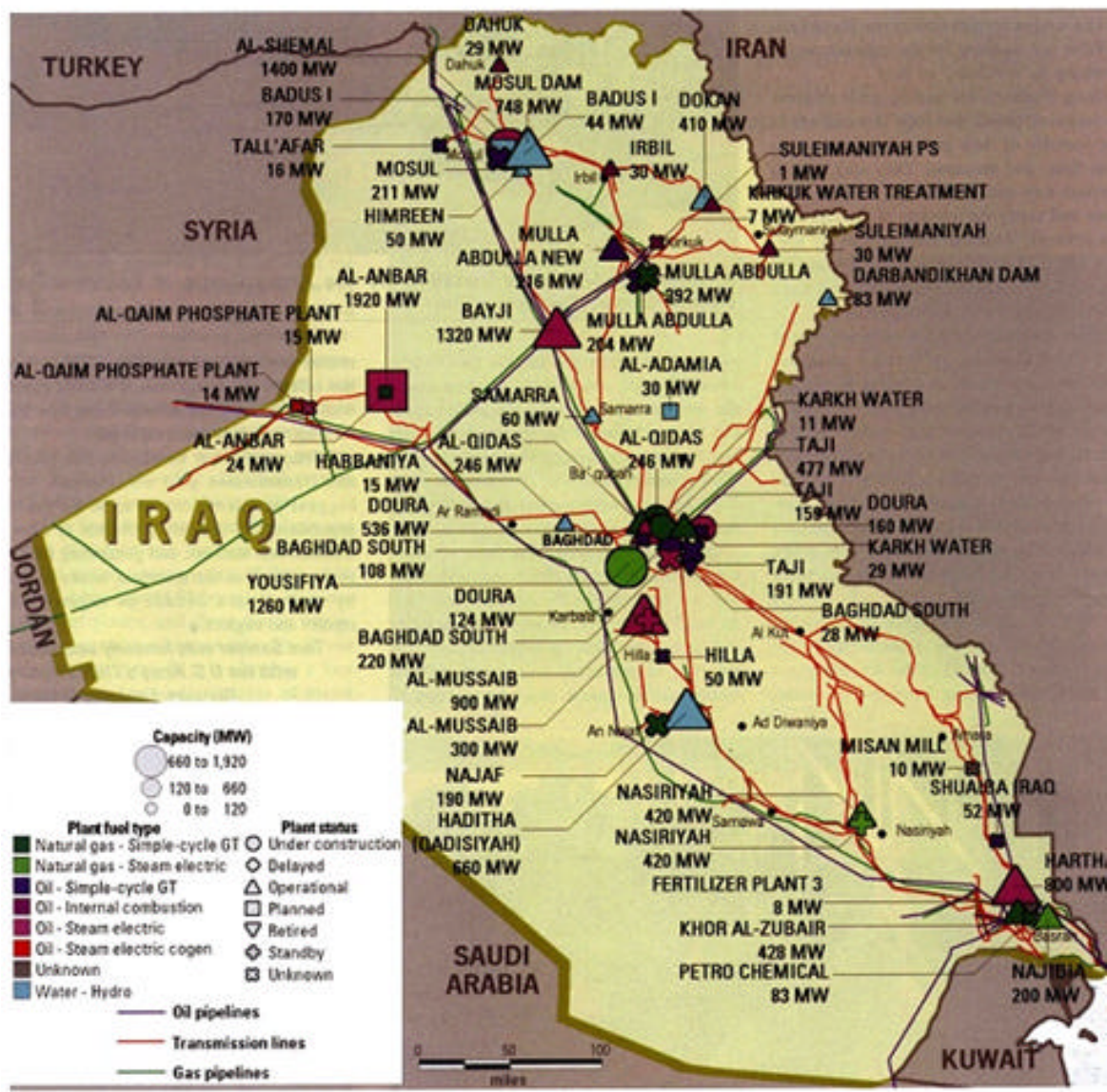

Source: Platts POWERTap

Fig. 1: Iraq power infrastructures

un acceptably low unless a conductor temperature well into the annealing range is used. Since, the most severe ambient conditions occur infrequently, it is customary to select less severe ambient conditions to produce higher ratings. In attempt to keep the estimated loss of strength with in tolerable limits, ambient conditions and maximum conductor temperature are usually chosen conservatively. There is however, a possibility of exceeding the allowable temperature whenever more severe ambient condition occur [EPRI2005] (Fig. 1):

\section{MATERIALS AND METHODS}

Maximum conductor temperature: Elements that oversee the maximum permissible functional temperature of transmission line are:

- The minimum permissible crushed clearance

- The capability of the fittings to function satisfactorily

- The satisfactory loss of the strength
The National Electric Security Code needs the minimum ground clearances be planned using the highest conductor functioning temperature, regardless of period. Thus, the increase in drop due to the elongation of the conductor must be careful for the highest electrode operating temperature. Process at high temperatures may touch the tensile strength of aluminum electrode.

Experience to these high temperatures may reason cumulative fractional annealing which will, in period, lower the strength of aluminum electrodes. The resulting damage of strength should be careful for temperatures overhead the $90-100^{\circ} \mathrm{C}$ range

Loss of strength in above conductors: Damage of strength is one issue to be considered when defining the maximum functioning temperature of a transmission link conductor. Equations may be used to control the loss of strength due to raised conductor temperatures. The exactness of these controls is dependent on predicting the number of hours the conductor will be functioned at temperatures greater than or equivalent to $100^{\circ} \mathrm{C}$. Damage of strength is 


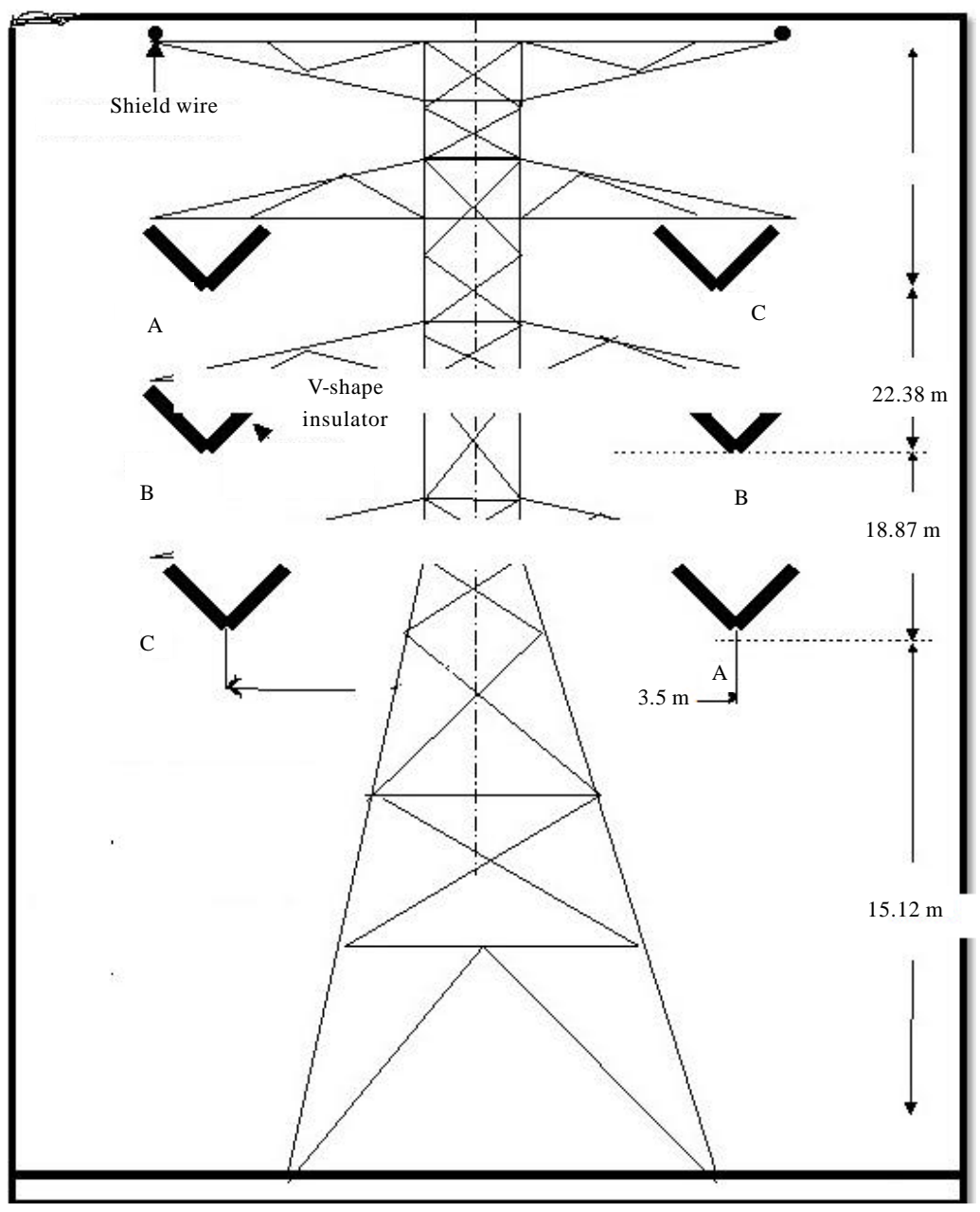

Fig. 2: Double circuit vertical configuration tower

cumulative during the life of the conductor. The guideline can be used to select a maximum operating temperature which would boundary the damage of strength to $<10 \%$ of its rated flouting strength over the lifetime of the conductor. This value of $10 \%$ damage of strength also looks in several conductor manufacturers works as a design value for upstairs lines.

\section{RESULTS AND DISCUSSION}

High voltage power line in Iraq: Figure 1 displays the power networks allocate electricity generated at the power place via. high voltage lines. In parts where power wants to be dispersed to consumers, transformers are rummage sale to change this high voltage into a abundant inferior voltage at the substation. Before incoming the customer's household or buildings, another transformer is used to drip the voltage depressed to more manageable stages. Usually power networks transmit electricity in three stages of voltages, Great Voltage (GV), Average Voltage (AV) and Little Voltage (LV). The variety of voltages is as in Fig. 2.

Acclimate and temperature: The acclimate bend of a conductor which is crossed between two points is contingent on the space between the stable points and the tension in the electrode and can be stated by the funicular bend. The always obtainable wire tension is mutable by the wire temperature and the extra load. This enduring stress clues to an irreparable elongation.

The design volume of a transmission line is designed by creating molds about the ambient temperature, breeze speed and other weather linked variables. The design working temperature is that when the acclimate is extra than that obligatory to encounter the statutory ground permission under these design assumptions. Certain of the variables that touch the conductor acclimate contain weather conditions, cross unit of the conductor and its 

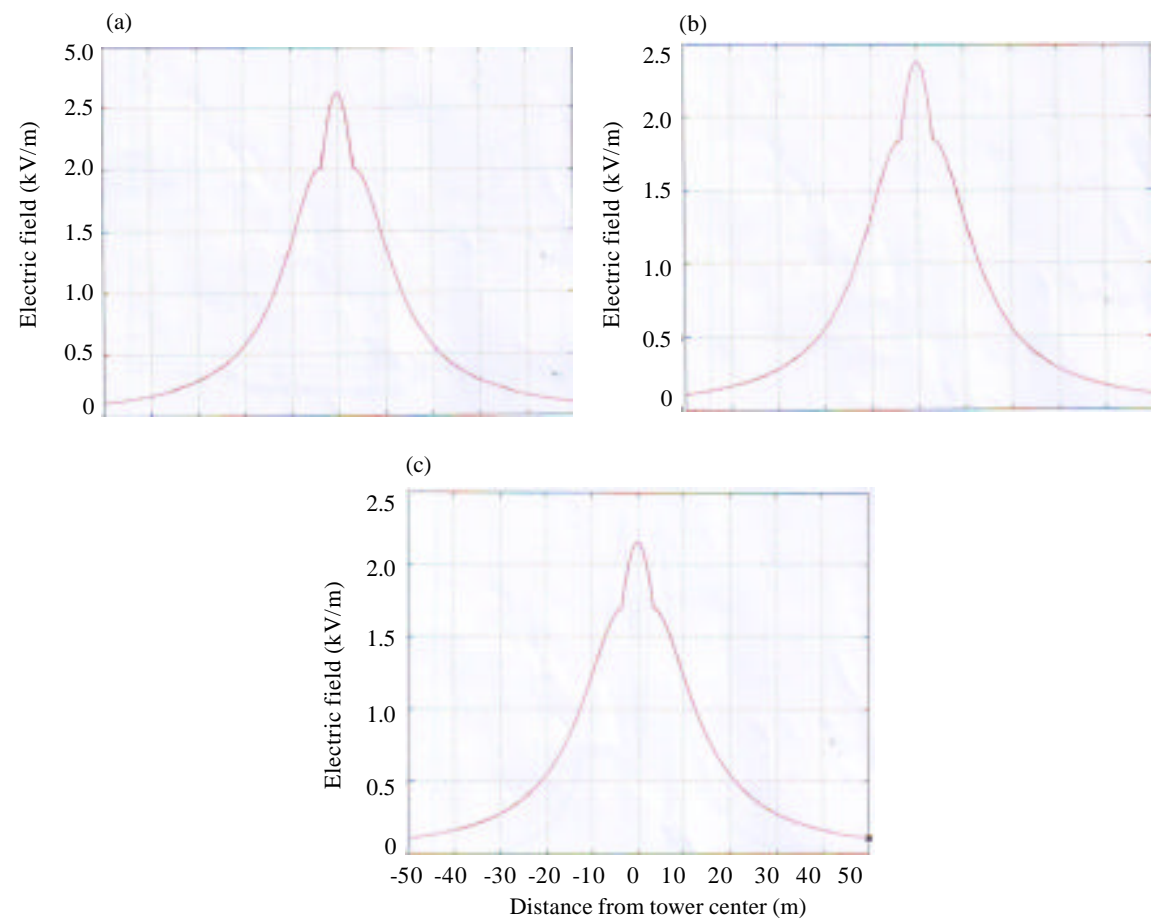

Fig. 3: a-c) Group 4 (case 1-3) generated results for electrical field densities at different heights $(8.86,8.3 .7 .76)$
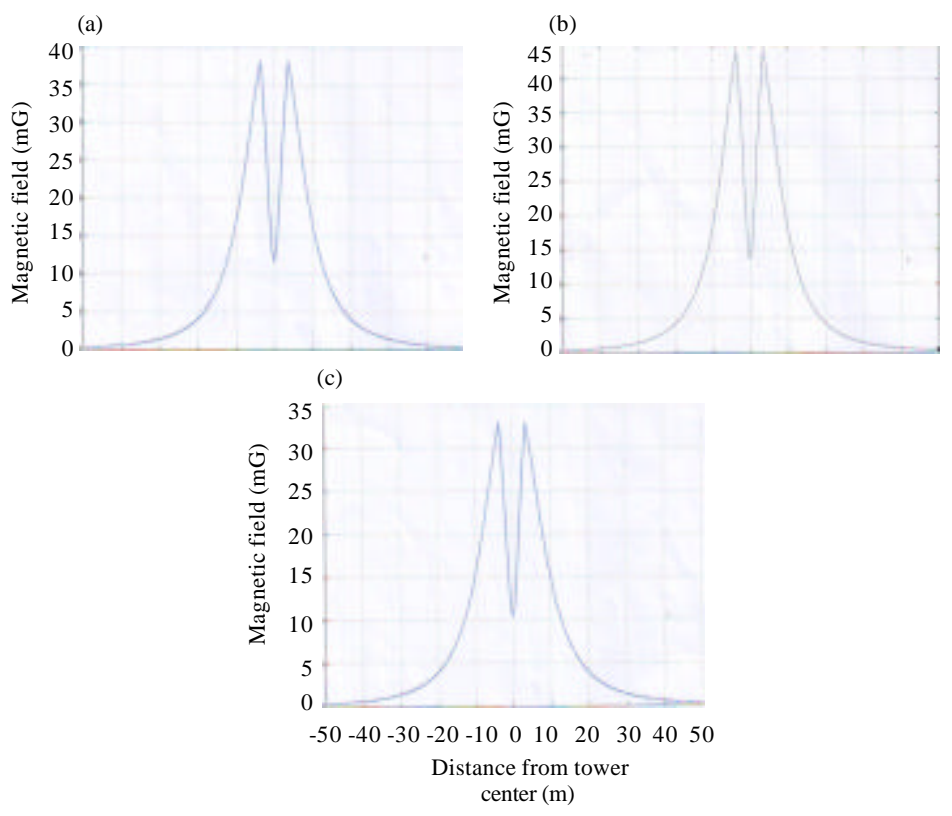

Fig. 4: a-c) Case 1-3 generated results for magnetic field densities at different heights $(8.86,8.3 .7 .76)$

manufacturer's specifications. To withstand these belongings the line is typically designed with design safety limits and can operate dependably and safely as long as the line acclimate does not disrupt the design bounds (Fig. 3 and 4).
The calculated magnetic fields: In this study the calculation of magnetic fields generated in the vicinity of high voltage transmission line was done. The MATLAB modules has been used to calculate the magnetic fields as a specific height above the ground level 
corresponding to instant voltage and currents and then to calculated the maximum value of effective fields at:

- Voltage level is $132 \mathrm{kV}$ line to line

- Line current $250 \mathrm{~A}$

- Vertical configuration is considered

- Conductors at height $(7.76,8.3,8.86 \mathrm{~m})$, respectively from ground level

- Horizontal separation between each two adjusted phase is $(3.4 \mathrm{~m})$

- Vertical separation between each two adjusted phase is $(1.3 \mathrm{~m})$

- Three different heights (h) above the ground level are studied and used in calculating process

Figure 3 (case 1-3) generated results for electrical field densities at different heights $(8.86,8.3 .7 .76)$, respectively above ground level for three-phase double circuit vertical configurations under investigation. Figure 4 group 5 (Fig. 1-3) generated results for magnetic field densities at different heights $(8.86,8.3 .7 .76)$, respectively above ground level for three-phase double circuit vertical configurations under investigation reveals that, close to the tower center line, the generated density increase with decrease of ground clearance level, i.e., when we get closer to the location of maximum acclimate. It is also revealed in this figure that the maximum value of the generated magnetic field density is highly depended on the ground clearance level which indeed depended on conductor's temperature.

\section{CONCLUSION}

In this research we demonstrate effect of changing ambient temperature on the intensity of the electromagnetic field generated near over head high voltage power transmission lines $(132 \mathrm{kV})$ South of Mosul city (Iraq), by measuring the increase in conductor length between two consecutive towers. At higher ambient temperatures conductor length increase which leads acclimate to increasing, i.e., ground clearance decrease and thereby increase the intensity of the electromagnetic field leads to decreasing through safe corridor in the vicinity of the towers and that increase the rate of the risk of that disease been mentioned by ICNIRP for people who live near it.
The measurement of acclimate has been done by using advanced surveying device to monitor the conductor in the different seasons of the year where a temperature varies. Using superposition method with Mat lab to calculate and draw an electromagnetic field around the tower at selected temperatures. In the set of measurement conductor length change from $(30-80 \mathrm{~cm})$ and magnetic field intensity changes from (33-44 mG) due to temperature changes rang, the designed magnetic and electric field stages are matched to exposure guidelines assumed by International Commission on Non-Ionizing Radiation Protection (ICNIRP) to evaluate the risks.

It has been noticed that the higher the ambient temperature around conductor, the more intense electromagnetic field Which in turn leads to the lack of safe pasacclimatee this at constant load. As also noted, the less distance between two consecutive towers (SPAN) the greater the acclimate. So, these observations should be taken in account when design power transmissions line in those areas.

\section{REFERENCES}

Albizu, I., E. Fernandez, A.J. Mazon and J. Bengoechea, 2011. Influence of the conductor temperature error on the overhead line ampacity monitoring systems. IET. Gener. Transm. Distrib., 5: 440-447.

Anonymous, 1988. Conductor temperature research. Georgia Institute of Technology, Atlanta, Georgia.

Anonymous, 2015. Dynamic line rating for overhead lines-V6. European Network of Transmission, Brussels, Belgium.

Morgan, V.T., 1982. The thermal rating of overhead-line conductors Part I. The steady-state thermal model. Electr. Power Syst. Res., 5: 119-139.

Muhammad, R.H., 2011. Power Electronics Handbook. 3rd Edn., Elsevier, New York, USA., ISBN:978-0-12-382036-5, Pages: 1375.

Pavlinic, A. and V. Komen, 2017. Direct monitoring methods of overhead line conductor temperature. Eng. Rev., 37: 134-146.

Portier, C.J. and M.S. Wolfe, 1998. Assessment of health effects from exposure to power-line frequency electric and magnetic fields. Master Thesis, National Institute of Environmental Health Sciences, North Carolina, USA. 